FERMILAB-PUB-93/397-T

December, 1993

Submitted to Phys. Rev. D

\title{
Top Production: Sensitivity to New Physics
}

\author{
Christopher T. Hill and Stephen J. Parke \\ Fermi National Accelerator Laboratory \\ P.O. Box 500, Batavia, Illinois, 60510 円
}

\begin{abstract}
The production cross-section and distributions of the top quark are sensitive to new physics, e.g., the $t \bar{t}$ system can be a probe of new resonances or gauge bosons that are strongly coupled to the top quark, in analogy to Drell-Yan production. The existence of such new physics is expected in dynamical electroweak symmetry breaking schemes, and associated with the large mass of the top quark. The total top production cross-section can be more than doubled, and distributions significantly distorted with a chosen scale of new physics of $\sim 1 \mathrm{TeV}$ in the vector color singlet or octet $s$-channel. New resonance physics is most readily discernible in the high $-p_{T}$ distributions of the single top quark and of the $W$ boson.
\end{abstract}

\footnotetext{
${ }^{1}$ Electronic addresses: (internet) hill@fnal.fnal.gov, parke@fnal.fnal.gov.
} 


\section{Introduction}

The top quark has proven to be very much heavier than most people anticipated over a decade ago, and is now the only expected sequential fermion with a mass of order the electroweak symmetry breaking scale. Since all fermion Dirac masses in the standard model must necessarily arise from the very mechanism that breaks electroweak symmetry, the top quark is the entity, whose existence is assured, that is most strongly coupled to the symmetry breaking dynamics. The top quark may therefore prove to be a powerful probe of electroweak symmetry breaking physics.

Through the top quark we can imagine three ways in which new physics begins to make an appearance [1]:

(i) oblique radiative corrections, as in the comparison of $m_{t}$ to $M_{W}$ or $\sin ^{2} \theta$, etc., may yield inconsistencies in high statistics, precision tests;

(ii) the search for exotic decay modes, such as $t \rightarrow H^{+}+b$, may yield the first observation of scalars as in multiple Higgs doublet schemes or some of the plethora of SUSY particles;

(iii) the production distributions of top are potentially sensitive to exotic intermediate heavy states, such as new resonances or gauge bosons, which may favor strong coupling to the top quark.

In this paper we focus on the latter issue, since it will be easiest to place some interesting new limits immediately upon the discovery of top, rather than through the other programatic modes which require high statistics. The essential idea here is 
to view the $t \bar{t}$ system as a replacement for the $\mu \bar{\mu}$ system in Drell-Yan-like processes, with the motivation that heavy top may act as a special probe of new associated electroweak symmetry breaking physics.

Several candidate new physics contributions that might significantly modify top production come to mind. These include particles of extended technicolor [2] such as color singlet scalars and color nonsinglet resonances, (into which we could include generalized Higgs bosons), new color singlet vector mesons (which include new $U(1)$ 's, new $S U(2)$ 's, and "techni- $\rho$ 's"), possible large induced effects in walking extended technicolor [3], such as strongly coupled extended massive techni-bosons, and scalar SUSY partners. Many of these are expected to be reasonably strongly coupled to the top quark. For example, walking ETC requires that some special strong dynamics in the ETC sector be provided to generate the top quark mass [3]. Top quark condensation is the extreme limit in which new dynamics generates the top quark mass, simultaneously breaking the electroweak symmetry [4, which may also imply the existence of new strong dynamics at the TeV scale [5].

At $\sqrt{s}=1.8 \mathrm{TeV}$, top quark production is dominated by quark-anti-quark annihilation in a $p \bar{p}$ machine [ [6]. The actual cross-section increases over the next-to-leading order approximation by about 30\% when soft-gluon radiation effects are summed [7]. Thus, we can only say with reasonable confidence that the overall cross-section is known to about $\pm 50 \%$ in QCD. The kinematic distributions should be reliable, however, up to their overall normalization since they involve very large $p_{T}$, and are known to be reliably determined in other processes.

Our present strategy is two-fold. We first take a general approach, e.g., as in 
ref. [8], writing down a complete set of allowed $d=5$ and $d=6$ operator contact terms, subject to the relevant symmetries, and include their effects into top quark production. This is the usual approach one takes in considering new physics possibilities from a bottom-up perspective, and we find it to be instructive. The operator basis turns out to be severely restricted by electroweak and custodial chiral symmetry, $S U(2)_{L} \times S U(2)_{R}$, in the light sector. We show that large effects can occur at the Tevatron for contact terms which are additive, incoherent color singlet $s$-channel operators, or which add coherently to the $q \bar{q}$ annihilation amplitude, involving a color-octet $s$-channel. The contact terms yield approximately only an overall renormalization of total cross-section, and the kinematic distributions are not significantly distorted. A signal of distortion requires the nontrivial propagator poles of new resonances.

We then discuss the actual dynamics of hypothetical new resonances. Motivated by our contact term analysis, we consider color singlet and color-octet vector states. We first describe these resonances generally, and then specialize to particular models, e.g., one in which new octet vector resonances are massive gauge bosons. With resonances we see that dramatic distortions of the kinematic production distributions can occur, and we identify the top quark $p_{T}$ distribution and the decay $W$ boson $p_{T}$ distribution as sensitive observables. These distributions can distinguish between the various cases, e.g., color singlet vs. color octet, etc. We will not provide an encyclopedic study of all possibile masses and couplings of new resonances, but rather we will illustrate some generic cases which we believe are most representative. The key point that production studies of top may reveal new signals, and that limits will 
immediately emerge with the discovery of top, will be illustrated. Remarkably, we find that we will already be sensitive to the $\sim 1 \mathrm{TeV}$ scale with $\sim 100$ top pairs in hand.

\section{Contact Terms}

The problem of classifying the allowed $d \leq 6$ contact terms is equivalent to the problem of specifying the relevant symmetries that apply. We are interested in operators that mediate transitions between the light quarks $(u, d)$, as well as the gluon $G_{\mu \nu}^{a}$, and the heavy top quark, or third generation. We are also treating the electroweak theory in the broken phase and ignoring weak transitions. As such, it is appropriate to assume that the following symmetries are linearly realized: (i) all local gauge symmetries $S U(2)_{L} \times U(1) \times S U(3)$ apply linearly to the light quarks; (ii) custodial $S U(2)_{R}$ applies to the light fermions; (iii) we treat the top quark as a sterile singlet under $S U(2)_{L} \times S U(2)_{R}$; (iv) electric $U(1)$ invariance applies to all fields. Essentially, (i) and (ii) is a massless or chiral limit for all of the light fermions in the electroweak theory, i.e., this would be the exact global symmetry of the standard model if the light quark and leptons have vanishing masses. Conditions (iii) and (iv) reflect the fact that we are interested in the broken phase of the electroweak theory (but not electrodynamics) and the top quark has a mass of order the weak scale.

Thus, we organize the light quarks into $S U(2)_{L} \times S U(2)_{R}$ doublets as $\psi_{L}=(u, d)_{L}$ and $\psi_{R}=(u, d)_{R}$. The $d=6$ four-fermion operators may be written down upon considering the possible quantum numbers of new hypothetical force carriers, and we indicate the quantum numbers of the force carriers in $(S U(2), S U(3))$ notation. The 
symmetry assumptions powerfully restrict the $d=6$ four-fermion operators to the following:

Singlet $s$-channel, $(1,1)$ :

$$
\mathcal{O}_{L, R}^{1}=\left(\bar{\psi}_{L, R} \gamma_{\mu} \psi_{L, R}\right)\left(\bar{t} \gamma^{\mu} t\right) ; \quad \mathcal{O}_{L, R}^{2}=\left(\bar{\psi}_{L, R} \gamma_{\mu} \psi_{L, R}\right)\left(\bar{t} \gamma^{\mu} \gamma^{5} t\right)
$$

Flavor singlet, color-octet, $s$-channel, $(1,8)$ :

$$
\mathcal{O}_{L, R}^{3}=\left(\bar{\psi}_{L, R} \gamma_{\mu} \frac{\lambda^{a}}{2} \psi_{L, R}\right)\left(\bar{t} \gamma^{\mu} \frac{\lambda^{a}}{2} t\right) ; \quad \mathcal{O}_{L, R}^{4}=\left(\bar{\psi}_{L, R} \gamma_{\mu} \frac{\lambda^{a}}{2} \psi_{L, R}\right)\left(\bar{t} \gamma^{\mu} \gamma^{5} \frac{\lambda^{a}}{2} t\right)
$$

Here the flavor indices and fermion color indices are contracted within the parentheses, unless otherwise specifically noted. Note that the $S U(2)_{L} \times S U(2)_{R}$ linear chiral symmetry precludes all but current-current interactions (e.g., an operator such as $\left(\bar{\psi}_{L} \psi_{R}\right)(\bar{t} t)$ cannot be singlet under $\left.S U(2)_{L} \times S U(2)_{R}\right)$. This is really a statement of custodial $S U(2)$ protectionism, e.g., an operator of the form $\bar{q} q \bar{t} t$ would threaten to induce a large mass for $q$ via the induced $\langle 0|\bar{t} t| 0\rangle \sim m_{t} v^{2}$, where $v$ is the electroweak scale, and is thus disallowed by $S U(2)_{L} \times S U(2)_{R}$. Such operators could occur in reality, but would be suppressed by a power of $m_{q}$ which we take to be small.

There are many other $d=6$ operators we can write down which are consistent with the symmetries, but are all reducible by Fierz rearrangement back into the basis $\mathcal{O}_{L, R}^{1}$ through $\mathcal{O}_{L, R}^{4}$. The additional operators are as follows: 
$s$-channel, $\left(\frac{1}{2}, 1\right) ; s$-channel, $\left(\frac{1}{2}, 8\right)$ :

$$
\mathcal{O}_{L, R}^{5}=\left(\bar{\psi}_{L, R}^{i} \Gamma_{(x)}^{A} t\right)\left(\bar{t} \Gamma^{A(x)} \psi_{i, L, R}\right) ; \quad \mathcal{O}_{L, R}^{6}=\left(\bar{\psi}_{L, R}^{i} \Gamma_{(x)}^{A} \frac{\lambda^{a}}{2} t\right)\left(\bar{t}^{\lambda^{a}} \frac{\Gamma^{A(x)}}{2} \psi_{i, L, R}\right)
$$

The $\Gamma_{(x)}^{A}$ are generic Dirac matrices where $(x)$ represents the summed Lorentz indices and $A$ is an unsummed type label designating scalar $(A \equiv S)$, vector $(A \equiv V)$, tensor ( $A \equiv T)$, axial-vector $(A \equiv A)$, and pseudoscalar $(A \equiv P)$.

We furthermore note that $\psi$ may be replaced by its electroweak and Dirac chargeconjugate $\psi^{C}=i \tau^{2} C \bar{\psi}^{T}$, where $C=i \gamma^{0} \gamma^{2}$. This replacement must be done pairwise since we want to conserve $U(1)_{E M}$, and we note that the following operators can also be reduced back to the original basis $\mathcal{O}_{L, R}^{1}$ through $\mathcal{O}_{L, R}^{4}$ :

$t$-channel, $\left(\frac{1}{2}, \overline{3}\right) ; t$-channel, $\left(\frac{1}{2}, \mathbf{6}\right)$ :

$\mathcal{O}_{L, R}^{7}=\left(\bar{\psi}_{L, R[\alpha}^{C i} \Gamma_{(x)}^{A} t_{\beta]}\right)\left(\bar{t}^{[\alpha} \Gamma^{A(x)} \psi_{i, L, R}^{C \beta]}\right) ; \quad \mathcal{O}_{L, R}^{8}=\left(\bar{\psi}_{L, R\{\alpha}^{C i} \Gamma_{(x)}^{A} t_{\beta\}}\right)\left(\bar{t}^{\{\alpha} \Gamma^{A(x)} \psi_{i, L, R}^{C \beta\}}\right)$

where $\alpha$ and $\beta$ are color indices, appropriately symmetrized or antisymmetrized.

To see that $\mathcal{O}_{L, R}^{5}$ through $\mathcal{O}_{L, R}^{8}$ are reducible to $\mathcal{O}_{L, R}^{1}$ through $\mathcal{O}_{L, R}^{4}$, we first observe that operators like $\mathcal{O}_{L, R}=\left(\bar{\psi}_{L, R}^{i} \Gamma_{A} t\right)\left(\bar{t} \gamma^{5} \Gamma^{A} \psi_{i, L, R}\right)$ are equivalent to the $\mathcal{O}_{L, R}^{5}$ set upon commuting $\gamma^{5}$ through $\Gamma^{A}$, and using the chiral projections (similarly for the color-octet analogues). Also, we need only consider as independent the $S, V$, and $T$ combinations, since $A$ and $P$ are equivalent by the same argument. Moreover, the operators are not independent under Fierz rearrangement. For example, the $L L$ 
tensor operators vanish by chirality (similarly for $R R$ ):

$$
\begin{aligned}
\left(\bar{\psi}_{L}^{i \alpha} \sigma_{\mu \nu} t_{\alpha}\right)\left(\bar{t}^{\beta} \sigma^{\mu \nu} \psi_{i, \beta ; L}\right)= & -3\left(\bar{\psi}_{L}^{i \alpha} \psi_{i, \beta ; L}\right)\left(\bar{t}^{\beta} t_{\alpha}\right)-\frac{1}{2}\left(\bar{\psi}_{L}^{i \alpha} \sigma_{\mu \nu} \psi_{i, \beta ; L}\right)\left(\bar{t}^{\beta} \sigma^{\mu \nu} t_{\alpha}\right) \\
& \left.-3\left(\bar{\psi}_{L}^{i \alpha} \psi_{i, \beta ; L}\right)\left(\bar{t}^{\beta} \gamma^{5} t_{\alpha}\right)\right) \\
= & 0
\end{aligned}
$$

The remaining $S$ and $V$ can be related to $\mathcal{O}_{L, R}^{1}$ through $\mathcal{O}_{L, R}^{4}$, e.g., as in

$$
\left(\bar{\psi}_{L, R}^{i} t\right)\left(\bar{t} \psi_{L, R}^{i}\right)=-\frac{1}{2}\left(\bar{\psi}_{L, R} \gamma_{\mu} \frac{\lambda^{a}}{2} \psi_{L, R} \bar{t} \gamma^{\mu} \frac{\lambda^{a}}{2} t \pm \bar{\psi}_{L, R} \gamma_{\mu} \frac{\lambda^{a}}{2} \psi_{L, R} \bar{t} \gamma^{\mu} \gamma^{5} \frac{\lambda^{a}}{2} t+\mathcal{O}\left(1 / N_{c}\right)\right)
$$

with the $+(-)$ sign for the $L L(R R)$ case (keeping terms beyond leading order in $1 / N_{c}$ does not affect the argument).

In the case of the operators $\mathcal{O}_{L, R}^{7}$ and $\mathcal{O}_{L, R}^{8}$, the color of $\psi^{C}$ is conjugated, which leads to color $t$-channels $\overline{\mathbf{3}}$ or $\mathbf{6}$. However, the same Fierz reduction arguments apply to this set as before. We can always treat $\psi$ as a generic two-component electroweak doublet, Dirac field and at the end of the rearrangement replace it by $\psi^{C}$. Thus, in the case of $O^{7}$, we will obtain by Fierz rearrangement:

$$
\begin{aligned}
O^{7} & \rightarrow\left(\bar{\psi}_{[\alpha}^{C} \Gamma^{A} \psi^{C \beta]}\right)\left(\bar{t}^{[\alpha} \Gamma^{A} t_{\beta]}\right) \rightarrow\left(\bar{\psi}^{C} \gamma_{\mu}\left(\frac{\lambda^{A}}{2}\right)^{T} \psi^{C}\right)\left(\bar{t} \gamma^{\mu} \frac{\lambda^{A}}{2} t\right)+\ldots \\
& \rightarrow\left(\bar{\psi} \gamma_{\mu} \frac{\lambda^{A}}{2} \psi\right)\left(\bar{t} \gamma^{\mu} \frac{\lambda^{A}}{2} t\right)+\ldots
\end{aligned}
$$

and this set is also equivalent to $\mathcal{O}_{L, R}^{1}$ through $\mathcal{O}_{L, R}^{4}$. Physically, in $q \bar{q}$ annihilation we have only the possibility of $s$-channel singlet and octet; $t$-channel $\overline{\mathbf{3}}$ and $\mathbf{6}$ exchange is indistinguishable from $s$-channel singlet and octet exchange at low energies. Thus, the combination of light quark electroweak and custodial symmetries together with 
the top treated as an effective sterile singlet yields a large reduction of the operator basis that we would have to consider otherwise.

There are, in addition, the $d \leq 6$ gluonic operators,

$$
\begin{aligned}
\mathcal{O}_{g}^{1}=m_{t} G_{\mu \nu}^{a} \bar{t} \sigma^{\mu \nu} \lambda^{a} t ; & \mathcal{O}_{g}^{2}=m_{t} \tilde{G}_{\mu \nu}^{a} \bar{t} \sigma^{\mu \nu} \lambda^{a} t ; \\
\mathcal{O}_{g}^{3}=\left(D^{\mu} G_{\mu \nu}\right)^{a} \bar{t} \gamma^{\nu} \lambda^{a} t ; & \mathcal{O}_{g}^{4}=\left(D^{\mu} G_{\mu \nu}\right)^{a} \bar{t} \gamma^{\nu} \gamma^{5} \lambda^{a} t ;
\end{aligned}
$$

$\mathcal{O}_{g}^{3}$ and $\mathcal{O}_{g}^{4}$ are penguin-like and equivalent by use of equations of motion to the four-fermion operator set. $d=5$ operators of this form might be expected from strong-ETC radiative corrections to the top-top-gluon vertex. We therefore include a factor of $m_{t}$ in their definition, which is the natural scale of these operators (with an arbitrary $\frac{1}{\Lambda}$ coefficient in the effective Lagrangian we would end up with unnaturally large contributions of the effects of these terms; if they are large in production dynamics, then they would also be expected to produce a large induced $m_{t}$ as well). At the Tevatron we find that there is little sensitivity to the gluonic contact terms from the subprocess $g+g \rightarrow \bar{t}+t$ in the top quark production rate. The gluonic operators can contribute to the top quark production in the subprocess $\bar{q}+q \rightarrow \bar{t}+t$. However the extra factors of $m_{t}$ suppress these contributions compared with the previous four fermion operators. Previous authors have considered the effect of the color-octet pseudo-Nambu-Goldstone boson [9, 10] and anomalous gluonic magnetic moment operators (as described below) in $\bar{t} t$ production. However, these effects were considered only in the gluon fusion subprocess, i.e., the leading process in a $p p$ machine such as SSC or LHC. In general these gluon fusion processes are a small effect for us. We shall not consider them further presently, and refer the reader to ref. [9] and ref. [10]. 
The color singlet $s$-channel operators give incoherent additive corrections to the QCD annihilation cross-section. On the other hand, color-octet $s$-channel operators must be added coherently to the single gluon annihilation amplitude in $\bar{q}+q \rightarrow \bar{t}+t$. Let us consider the particular effective interaction Lagrangians:

$$
\mathcal{L}_{1}^{\prime}=\frac{g_{3}^{2}}{\Lambda^{2}}\left(\mathcal{O}_{L}^{1}+\mathcal{O}_{R}^{1}\right) \quad \mathcal{L}_{2}^{\prime}=\frac{g_{3}^{2}}{\Lambda^{2}}\left(\mathcal{O}_{L}^{3}+\mathcal{O}_{R}^{3}\right)
$$

where we include a factor of the QCD coupling constant, $g_{3}^{2}$, which will be seen to be convenient when we compare to actual resonance models as described in the next section $\left(g_{3}^{2}\right.$ is a smaller normalization factor than used in ref.[8], who assume a strong coupling constant $g^{2} \sim 4 \pi$; we take typically $g_{3}^{2} \sim 4 \pi \times(0.11)$; therefore our scale $\Lambda$ will always be $\sim 1 / 3$ of the scale obtained by using the ref. [8 normalization). We have modified the standard tree level top production calculation [11] to include the contact terms in $\mathcal{L}_{i}^{\prime}$ and have used the HMRS Set-B parton distribution functions [12] with both the factorization and renormalization scales set equal to $m_{t} / 2$.

We see in Figures 1(a) and 1(b) the results for the ratio of the total top production cross-section, to the QCD cross-section, with various scales $\Lambda$ (thus $R=1$ is $\Lambda \rightarrow \infty$, or pure QCD). We have plotted these as functions of $m_{t}$. The effects of the singlet operators are shown in Fig. 1(a), while octets are shown in Fig. 1(b). With the signs chosen for the color octet contact terms of $\mathcal{L}_{2}^{\prime}$ we generate coherent additive enhancement of top production (denoted in Fig. 1(b) by + ); with the opposite sign (denoted - ) for the color-octet $s$-channel operators, i.e., $\mathcal{L}_{2}^{\prime} \rightarrow-\mathcal{L}_{2}^{\prime}$ we suppress the production cross-section. Clearly, the enhancement or suppression of the crosssection by the contact term effect increases with increasing $m_{t}$ for fixed $\Lambda$ because the gluon propagator is decreasing with increasing $m_{t}$ while the contact term remains 
constant.

In Fig.(2) we plot the differential distributions $d \sigma / d p_{T}$ for the $p_{T}$ of the single top quark system and the $p_{T}$ of the $W$ boson produced by a decaying top quark. The $W$ boson $p_{T}$ is readily known since the lepton and missing $p_{T}$ will be known in the $\ell+4 j$ sample. In the $\ell+4 j$ sample it should also be possible to cut the $3 j$ invariant mass near the top quark mass and determine the $3 j p_{T}$ to infer the distribution of the top quark with $p_{T}$. We have given these distributions because we have found that they are empirically the most sensitive to new resonance physics, as described below, thus optimizing the potential observability of new physics. However, we see that when the new physics is given only by a contact term, for fixed $m_{t}$, the QCD distributions are only approximately renormalized by an overall multiplier, i.e., no significant distortion of the distributions is seen. Thus, these distributions are shaped primarily by the phase space and kinematics. This is a problem for the observability of mere contact term effects, since one must trust the knowledge of the overall QCD production cross-section normalization. Nonetheless, if we discover that top is produced with a total cross-section that is significantly larger or smaller than the QCD predictions of refs. [6, 7], then it is possible that one is seeing the effects of

new physics. For example, if the scale of the contact term is $\Lambda \leq 600 \mathrm{GeV}$ then the total top cross-section is increased relative to pure QCD by a factor greater than 2 .

\section{A New Strong Dynamics}

The contact term analysis reveals a possible sizeable enhancement of top production through new color-singlet or color-octet $s$-channel vector mesons. This may 
involve either a new intermediate force carrier in the $s$-channel, or $t$-channel, $\overline{3}$ or 6 exchange, by Fierz rearrangement. The contact terms lead only to multiplicative renormalization of the kinematic distributions, and are therefore not easy to distinguish, except by their very large effects when $\Lambda \lesssim 0.6 \mathrm{TeV}$. We turn now to dynamical resonances which are generally expected to have influence in kinematic distributions.

A new color singlet $s$-channel resonance $B_{\mu}$ of mass $M_{B}$, can be either a boundstate, as in technicolor models, or a new gauge boson, such as a heavy $Z^{\prime}$ gauge field. For example, the model of Lindner and Ross [5] attempts to generate top condensation with such a field, and favors strong coupling to top. We will presently confine our attention to vector current couplings, in which case the coupling to the light quark doublets and the top quark can be taken as:

$$
g_{3}\left(\tilde{z}_{1} \bar{\psi} \gamma_{\mu} \psi+\tilde{z}_{2} \bar{t} \gamma_{\mu} t\right) B^{\mu}
$$

where $\tilde{z}_{1}$ and $\tilde{z}_{2}$ are just scale factors for the interaction strength relative to QCD (we use the ${ }^{\sim}$ for the color singlet case). The width of the singlet vector boson to decay to $n_{f}$ flavors of light quark pairs, and to $t \bar{t}$ in the massless approximation (for the $M_{B}$ values we consider the massless approximation is valid even for $m_{t} \sim 160 \mathrm{GeV}$ ) is:

$$
\Gamma_{B}=\frac{g_{3}^{2} M_{B}}{12 \pi}\left(n_{f} \tilde{z}_{1}^{2}+\tilde{z}_{2}^{2}\right)
$$

In general, the presence of the vector boson will modify the QCD production crosssection for the parton subprocess $\bar{q}+q \rightarrow \bar{t}+t$ as:

$$
\sigma_{\bar{q}+q \rightarrow \bar{t}+t}=\sigma_{Q C D}\left[1+C_{3} \frac{\tilde{z_{1}^{2}} \tilde{z}_{2}^{2} s^{2}}{\left[\left(s-M_{B}^{2}\right)^{2}+M_{B}^{2} \Gamma_{B}^{2}\right]}\right]
$$

where $C_{3}$ is an $S U(N)$ color factor given by $4 N^{2} /\left(N^{2}-1\right) \sim 4.5$. 
We also consider a color octet of massive vector bosons, $B_{\mu}^{A}$, also of mass $M_{B}$, which we will call "colorons." These are necessarily degenerate because QCD is unbroken. Such objects may be composite, $\rho$-like objects, with a scale of compositeness of order $\sim M_{B}$ which serves as a cut-off scale for the $B_{\mu}^{A}$ effective Lagrangian. It then suffices to consider $B_{\mu}^{A}$ as a linear representation of QCD (if $B_{\mu}^{A}$ are fundamental, with effectively infinite cut-off, then consistency requires they are necessarily gauge bosons, as we consider below). We can write a phenomenological coupling as:

$$
g_{3}\left(z_{1} \bar{\psi} \gamma_{\mu} \frac{\lambda^{A}}{2} \psi+z_{2} \bar{t} \gamma_{\mu} \frac{\lambda^{A}}{2} t\right) B^{A, \mu}
$$

Here we again use rescaled QCD couplings, $z_{1} g_{3}$ and $z_{2} g_{3}$. The process $\bar{q}+q \rightarrow$ $\bar{t}+t$ involves the coherent sum of the gluon and coloron $s$-channel amplitudes. It is therefore easy to implement the effect of the coloron in QCD production, i.e., we simply make the replacement in the gluon propagator:

$$
\frac{g_{3}^{2}}{s} \rightarrow \frac{g_{3}^{2}}{s}+\frac{g_{3}^{2} z_{1} z_{2}}{\left(s-M_{B}^{2}\right)+i M_{B} \Gamma_{B}}
$$

The production amplitude therefore depends upon the product of the new rescaled coupling constants, $z_{1} z_{2}$ (which can be negative), the mass $M_{B}$, and the coloron width $\Gamma_{B}$. As we will see below, the relative sign of these terms, determined by the sign of $z_{1} z_{2}$, has a significant effect in shaping the top quark $p_{T}$ distributions.

The width of the coloron to decay to $n_{f}$ flavors of light quark pairs, and to $t \bar{t}$ in the massless approximation (for the $M_{B}$ values we consider the massless approximation is valid even for $m_{t} \sim 160 \mathrm{GeV}$ ) is:

$$
\Gamma_{B}=\frac{g_{3}^{2} M_{B}}{24 \pi}\left(n_{f} z_{1}^{2}+z_{2}^{2}\right)
$$


Obviously, the width can be readily generalized. Thus, in the generic phenomenological model, top production depends upon 3 independent parameters, $z_{1} z_{2}, M_{B}$ and $\Gamma_{B} / M_{B} \sim\left(n_{f} z_{1}^{2}+z_{2}^{2}\right)$.

It is useful to reduce the parameters by focusing on a particular version of the general model which is dictated by simple additional symmetry assumptions. Thus, we turn to the case in which $B$ is a gauge boson. Let us consider a simple scheme in which the third generation participates in a new strong gauge dynamics, analogous to QCD. This has been previously suggested by the fact that the top quark is very heavy [5], implying either strong coupling to the electroweak breaking dynamics, or associated with that breaking through "top condensation." Our present model is a slightly simpler variation on ref. [5].

We assume a minimal extension of the standard model such that at scales $\mu \gg$ $M$, we have the gauge group $U(1) \times S U(2)_{L} \times S U(3)_{1} \times S U(3)_{2}$. The coupling constants (gauge fields) of $S U(3)_{1} \times S U(3)_{2}$ are now respectively $h_{1}$ and $h_{2}\left(A_{1 \mu}^{A}\right.$ and $\left.A_{2 \mu}^{A}\right)$. We assign the usual light quark and lepton fields to representations under $\left(S U(2)_{L}, S U(3)_{1}, S U(3)_{2}\right)$ as follows:

$$
\begin{aligned}
& (u, d)_{L},(c, s)_{L} \rightarrow(2,3,1) \quad u_{R}, d_{R}, c_{R}, s_{R}, \rightarrow(1,3,1) \\
& \left(\nu_{e}, e\right)_{L}, \quad\left(\nu_{\mu}, \mu\right)_{L}, \quad\left(\nu_{\tau}, \tau\right)_{L} \rightarrow(2,1,1) \quad e_{R}, \mu_{R}, \tau_{R},\left(\nu_{i R}\right) \rightarrow(1,1,1)
\end{aligned}
$$

while the top and bottom quarks are assigned:

$$
(t, b)_{L} \rightarrow(2,1,3) ; \quad t_{R}, b_{R} \rightarrow(1,1,3)
$$


This assignment is anomaly free.

We break the symmetry $S U(3)_{1} \times S U(3)_{2} \rightarrow S U(3)_{c}$ at the scale $M$. We can do so by introducing a scalar (Higgs) field $\Phi_{b^{\prime}}^{a}$ which transforms as $(1,3, \overline{3})$. By a general choice of the $\Phi$ potential a VEV develops: $\langle\Phi\rangle=\operatorname{diag}(M, M, M)$. breaking $S U(3)_{1} \times S U(3)_{2}$ to the massless QCD gauge group $S U(3)_{c}$ with gluons, $A_{\mu}^{A}$ and a residual global $S U(3)^{\prime}$ with degenerate, massive colorons, $B_{\mu}^{A}$. The gluon $\left(A_{\mu}^{A}\right)$ and coloron $\left(B_{\mu}^{A}\right)$ fields are then defined by:

$$
A_{1 \mu}^{A}=\cos \theta A_{\mu}^{A}-\sin \theta B_{\mu}^{A} \quad A_{2 \mu}^{A}=\sin \theta A_{\mu}^{A}+\cos \theta B_{\mu}^{A}
$$

where:

$$
h_{1} \cos \theta=g_{3} ; \quad h_{2} \sin \theta=g_{3}
$$

and thus:

$$
\tan \theta=h_{1} / h_{2} ; \quad \frac{1}{g_{3}^{2}}=\frac{1}{h_{1}^{2}}+\frac{1}{h_{2}^{2}}
$$

where $g_{3}$ is the QCD coupling constant at $M$. In what follows we envision $h_{2} \gg h_{1}$ and thus $\cot \theta \gg 1$ (perhaps to select the top quark direction for condensation). The mass of the degenerate octet of colorons is given by:

$$
M_{B}=\left(\sqrt{h_{1}^{2}+h_{2}^{2}}\right) M=\left(\frac{g_{3}}{\sin \theta \cos \theta}\right) M
$$

The usual QCD gluonic interactions are obtained for all quarks (including top and bottom) while the coloron interaction takes the form:

$$
\mathcal{L}^{\prime}=-\left[g_{3} \cot \theta\left(\bar{t} \gamma_{\mu} \frac{\lambda^{A}}{2} t+\bar{b} \gamma_{\mu} \frac{\lambda^{A}}{2} b\right)-g_{3} \tan \theta \sum_{i} \bar{q}_{i} \gamma_{\mu} \frac{\lambda^{A}}{2} q_{i}\right] B^{\mu A}
$$

where the sum extends over all other light quarks. The appearance of the $\cot \theta$ factor in the light quark sector and the $-\tan \theta$ in the heavy quark sector implies that the 
production amplitude for top via annihilation $\bar{q}+q \rightarrow \bar{t}+t$ is independent of $\theta$, except through the coloron width. Note that we have now reduced the number of free parameters from 3 to 2 , i.e., $\cot \theta$ and $M_{B}$, since now $z_{1} z_{2}=-1$. Again, top production involves the coherent sum of the gluon and coloron $s$-channel amplitudes:

$$
\frac{g_{3}^{2}}{s} \rightarrow \frac{g_{3}^{2}}{s}-\frac{g_{3}^{2}}{\left(s-M_{B}^{2}\right)+i M_{B} \Gamma_{B}}
$$

Now a relative minus sign between the coloron and gluon contributions is fixed implying that the cross-section is amplified for $s<M_{B}^{2}$ (neglecting the $B$ width), and suppressed for $s>M_{B}^{2}$. This model involves only the free parameters $\cot \theta$ and $M_{B}$, and the sensitivity to $\cot \theta$ appears only through the width of the coloron $B$.

The decay width of the coloron is dominated by $t \bar{t}$ for large $\cot \theta$ :

$$
\Gamma_{B}=\frac{g_{3}^{2} \cot ^{2} \theta}{24 \pi} M_{B}
$$

If top-condensation occurs, or if the coloron plays a role in inducing a large top quark mass through near critical coupling, then $\cot \theta$ is roughly determined. Criticality implies $g_{3} \cot \theta=h>>1$; for example, the Nambu-Jona-Lasinio value of $h$ sufficient to trigger condensation is $h^{2} N_{c} / 8 \pi \gtrsim 1$. This yields $h^{2}=g_{3}^{2} \cot ^{2} \theta \gtrsim 8 \pi / 3$, and applying this to the coloron decay width yields:

$$
\Gamma_{B} \gtrsim \frac{2}{9} M_{H} .
$$

(where the factor of 2 includes both $b$ and $t$ contributions). We use this latter result to define the width of both colorons and color-singlets in the following, taking $\Gamma_{B}=$ $0.2 M_{B}$, and thus fixing all parameters except $M_{B}$. 
We consider subsequently the following three cases: (A): the color-singlet vector resonance model as defined by eq.(10) in which $z_{1} z_{2}= \pm 1$ and where we take the width $\Gamma_{B}=0.2 M_{B}$ for definiteness; $(\mathbf{B})$ : the gauge color-octet vector resonance model as defined by eq.(22) in which $z_{1} z_{2}=-1$; (C): a hybrid model in which the parameters of the gauge color-octet vector resonance model are assumed, but we invert by hand the relative signs between the $\cot \theta$ and $\tan \theta \operatorname{terms}$ of eq.(22), i.e, $z_{1} z_{2}=+1$

In the sequence of figures, 4(a), (b) and (c), we present the differential distributions for the single top quark $p_{T}$ in the three models. Fig. 4(a) represents the incoherent additive contribution of the color singlet vector resonance to the cross-section, i.e., model (A). Notice the large additive coherent enhancement of model (B) for smaller $p_{T}$, as seen in Fig. 4(b), where the amplitudes add below the mass of the resonance, while in $4(\mathrm{c})$ we give the results for the hybrid model (C) in which we have reversed the relative signs of the couplings of the vector boson to light quarks and the top quark. Here we see an actual coherent suppression of the distributions for low $p_{T}$, followed by a characteristic sizeable enhancement at high- $p_{T}$.

In Fig.(5) we present the differential distributions for the $p_{T}$ of the $W$ boson coming from the weak decay of a single top. In either the $W$ or $t$ distributions of Figs.(4) through (5) one sees a significant signal by the distortion relative to QCD for resonances as heavy as $800 \mathrm{GeV}$. As the resonance mass $M_{B}$ is taken larger than 800 $\mathrm{GeV}$ the distortions rapidly go away, due to the finite energy reach of the machine, assumed to be $\sqrt{s}=1.8 \mathrm{TeV}$. Even with a few events in hand one may see, in principle, a large excess of high- $p_{T}$ top quarks if such resonances are present. 
Finally, since we have argued the similarities of this approach with Drell-Yan, in Fig.(6) we present the differential distributions for the mass of the $t \bar{t}$ pair, $M_{t t}$. Again, a significant signal of distortion relative to QCD for resonances as heavy as $700 \mathrm{GeV}$ is seen.

Our results have assumed essentially a process in which the product of resonance production and decay coupling contants is given by $g_{3}^{2}$, or $\left|z_{1} z_{2}\right|=1$ for our three models. This is in general a conservative guess, though it is forced in the special case of model (B), and we emphasize that the product of coupling constants could be significantly larger than this in reality, i.e., $g_{3}^{2}$ at the scale $\sim m_{t}$ is really quite perturbative, and new resonances could enter with much stronger nonperturbative coupling constants in principle. Even at the level we have assumed for these couplings, we see that we are very sensitive to the resonance profiles. In effect, when the resonance can be excited the pole of the propagator is becoming $\sim 1 / M_{B} \Gamma_{B}$, and the effective value of $\Lambda$ is now much smaller than $M_{B}$; since we take $\Gamma_{B}=0.2 M_{B}$ we get an effective $\Lambda \sim 0.44 M_{B}$ from the pole, which increases the cross-section in the vicinity of the pole by $\sim 25$ times. For example, in the singlet case we can readily see the effects of a $700 \mathrm{GeV}$ resonance in a substantial increase in the top quark $p_{T}$. This indicates that production studies of top with large statistics would be sensitive to more weakly coupled states of large mass than we have assumed, though the mass sensitivity drops rapidly to zero when $M_{B} \gtrsim \frac{1}{2} \sqrt{s}$, favoring increased collider energies. 


\section{Conclusions}

With the discovery of the top quark we are entering a new and previously unexplored realm of fermions that have masses of order the weak scale, and thus couplings to the dynamics of electroweak symmetry breaking that are $\gtrsim O(1)$. New physics associated with the top quark might therefore be expected to occur, and this can first show up in the physics of top production.

To model this situation, we have first considered generic contact terms. We think that the treatment of the $t$ quark as a singlet and the use of $S U(2)_{L} \times S U(2)_{R}$ to constrain the light quark sector is a new approach, valid in the broken phase of the electroweak symmetry, which leads to a dramatic simplification of the operator basis. We then study the effects of such contact terms in an effective Lagrangian. We find that large effects can occur for the color singlet or octet in the $s$-channel in $q \bar{q}$ annihilation (though physically, this can also come about from a $\overline{\mathbf{3}}$ or $\mathbf{6}$ exchange in the $t$-channel). A significantly enhanced or suppressed top production rate relative to QCD is indicative of new physics of this kind, and can occur for $\Lambda$ as large as $\Lambda \sim 0.7$ $\mathrm{TeV}$ (if we used the stronger coupling normalization of [8] we would be sensitive to $\Lambda \sim 2.0 \mathrm{TeV})$

We are therefore lead to consider models of specific strongly coupled vector resonances and gauge bosons, which have been previously motivated by consideration of the relationship of the top quark to electroweak symmetry breaking. We consider essentially three cases, (A) a color singlet vector resonance; (B) a gauge color octet vector resonance (a slight variation on ref. [5]), and (C) a hybrid color octet vector 
resonance in which the resonant amplitude has an inverted sign. We fix the resonance width by a strong coupling argument to be $\Gamma_{B} \sim 0.2 M_{B}$, and the product of (rescaled) couplings $\left|z_{1} z_{2}\right|=1$ (this happens automatically in model (B)), leaving only a remaining free parameter, the resonance mass, $M_{B}$. We study the top and $W$ $p_{T}$ distributions, and the $t \bar{t}$ mass distribution in the three models. These three models have characteristically different signatures. We emphasize that the results for observables that we present are not the most general, but are reasonable first guesses as to what might be expected if new strong dynamics is involved. The effects we generate can be dramatic, leading to significant enhancements of top production cross-sections for a scale of new physics of order $1 \mathrm{TeV}$, and to significant and observable distortions of the $p_{T}$ distributions of the $W$ boson and top system.

Qualitatively, we see that top quarks produced with large cross-section and excessively large $p_{T}$ would be indicative of the excitation of new resonances and may be observed with $\sim 100 t \bar{t}$ events in $\ell+4$ jets. A corollary is that the aplanarity of top events would be expected to be smaller, since the top system is boosted to larger $p_{T}$ than normally expected. These might be the best first order indicators of such new physics, and could conceiveably be seen with the first handful of top events.

Certainly a new strong dynamics for electroweak symmetry breaking in concert with the large value of $m_{t}$, must be taken seriously. Meaningful limits will be readily established with fewer than 100 top quarks in hand, and a non-null discovery potential is evident. With the discovery of the top quark we may be commencing the first observation of new physics beyond the standard model. 


\section{Acknowledgements}

We thank E. Eichten, and W. Giele for useful discussions. This work was performed at the Fermi National Accelerator Laboratory, which is operated by Universities Research Association, Inc., under contract DE-AC02-76CHO3000 with the U.S. Department of Energy. 


\section{Figure Captions}

Figures $1(\mathrm{a}), 1(\mathrm{~b})$ : The ratio of total cross-sections, $R=\sigma_{\text {contact }} / \sigma_{Q C D}$, for $t \bar{t}$ production including the effect of contact terms, relative to the QCD result, $R=1$. (a) is color singlet $s$-channel $\mathcal{L}_{1}^{\prime}$; (b) is color octet $s$-channel $\mathcal{L}_{2}^{\prime}$ with the coherently additive + or subtractive - signs.

Figure 2(a), 2(b) The differential distribution, $\frac{d \sigma}{d p_{T}}$, where $p_{T}$ is the transverse momentum of a single top quark. $m_{t}=160 \mathrm{GeV}$ is assumed, and results given for contact terms with $\Lambda=0.6 \mathrm{TeV}, \Lambda=0.8 \mathrm{TeV}$, and $\Lambda=\infty$ corresponding to pure QCD. (a) is color singlet $s$-channel $\mathcal{L}_{1}^{\prime}$; (b) is color octet $s$-channel additive $\mathcal{L}_{2}^{\prime}$.

Figure 3(a), 3(b): The differential distribution, $\frac{d \sigma}{d p_{T}}$, where $p_{T}$ is the transverse momentum of a $W$ boson from the weak decay of a single top quark. $m_{t}=160 \mathrm{GeV}$ is assumed, and results given as in Fig.(2).

Figure 4(a), 4(b), 4(c): The differential distributions, $\frac{d \sigma}{d p_{T}}$, where $p_{T}$ is the transverse momentum of a single top quark, $m_{t}=160 \mathrm{GeV}$, for the following sequence: (a) the gauge color-singlet vector resonance model (A); (b) the gauge color-octet vector resonance model $(\mathbf{B})\left(\right.$ with $z_{1} z_{2}=-1$ ); (c) the hybrid gauge color-octet vector resonance model $(\mathbf{C})$ (with $z_{1} z_{2}=+1$ ). Results are given in each case for resonance masses $M_{B}=(0.6,0.7,0.8,1.0) \mathrm{TeV}$, and $M_{B} \rightarrow \infty$ corresponding to pure QCD.

Figure 5(a), 5(b), 5(c): The differential distribution, $\frac{d \sigma}{d p_{T}}$, where $p_{T}$ is the transverse 
momentum of a $W$ boson from the weak decay of a single top quark. Results are presented in sequence as in Fig.(4).

Figure 6(a), 6(b), 6(c): The differential distribution, $\frac{d \sigma}{d M_{t t}}$, where $M_{t t}$ is mass of the $t \bar{t}$ quark pair. Results are presented in sequence as in Fig.(4). 


\section{References}

[1] C. T. Hill, High Luminosity Workshop, invited talk, Fermilab (Pheasant Run), Dec. 1 (1993).

[2] S. Dimopoulos, L. Susskind, Nucl. Phys. B155 237 (1979);

E. Eichten, K. Lane, Phys. Lett. 90B 125 (1980);

E. Eichten, I. Hinchliffe, K. Lane, C. Quigg, Phys. Rev. D34 1547 (1986);

[3] B. Holdom, Phys. Rev. Lett. 601223 (1988);

T. Appelquist, M. Einhorn, T. Takeuchi, L. C. R. Wijewardhana, Phys. Lett. B220 223 (1989). T. W. Appelquist, D. Karabali, L.C.R. Wijewardhana Phys. Rev. Lett. 57, 957 (1986);

[4] W. A. Bardeen, C. T. Hill, M. Lindner Phys. Rev. D41, 1647 (1990), and refs. therein.

[5] C. T. Hill, Phys. Lett. B266 419 (1991); (in our case the $b$ quark is treated symmetrically with $t$; to have top-condensation we can exploit the electroweak $U(1)$ interaction in concert with the colorons in generating the condensate, favoring $\langle\bar{t} t\rangle \neq 0$ while $\langle\bar{b} b\rangle=0$, with some fine-tuning; the $b$ quark mass can then be induced through the effect of coloronic instantons).

Interesting generalizations are given by: S. P. Martin, Phys. Rev. D46, 2197 (1992); Phys. Rev. D45, 4283 (1992);

For color singlet versions see: M. Lindner and D. Ross, Nucl. Phys. B 370, 30 
(1992); R. Bonisch, "Gauge Created Top Quark Condensate and Heavy Top," Munich University Preprint, LMU-91-03 (1991), unpublished.

[6] P. Nason, S. Dawson, R. K. Ellis, Nucl. Phys., B303, 607 (1988); Nucl. Phys., B327, 49 (1988); erratum, Nucl. Phys., B335, 260 (1990).

[7] E. Laenen, J. Smith, W. L. Van Neerven, "Top Quark Production Cross Section," Fermilab-Pub-93/270-T

[8] E. Eichten, K. Lane, M. Peskin, Phys. Rev. Lett. 50, (1983) 811.

[9] T. Appelquist and G. Triantaphyllou, Phys. Rev. Lett. 692750 (1992).

[10] D. Atwood, A. Aeppli, A. Soni, Phys. Rev. Lett. 692754 (1992).

[11] R. Kleiss and W. J. Stirling, Z. Phys. C40, 419 (1988).

[12] P. Harriman, A. Martin, R. Roberts and W. J. Stirling, Phys. Rev D42, 798 (1990). 
This figure "fig1-1.png" is available in "png" format from: http://arxiv.org/ps/hep-ph/9312324v1 
This figure "fig2-1.png" is available in "png" format from: http://arxiv.org/ps/hep-ph/9312324v1 
This figure "fig3-1.png" is available in "png" format from: http://arxiv.org/ps/hep-ph/9312324v1 


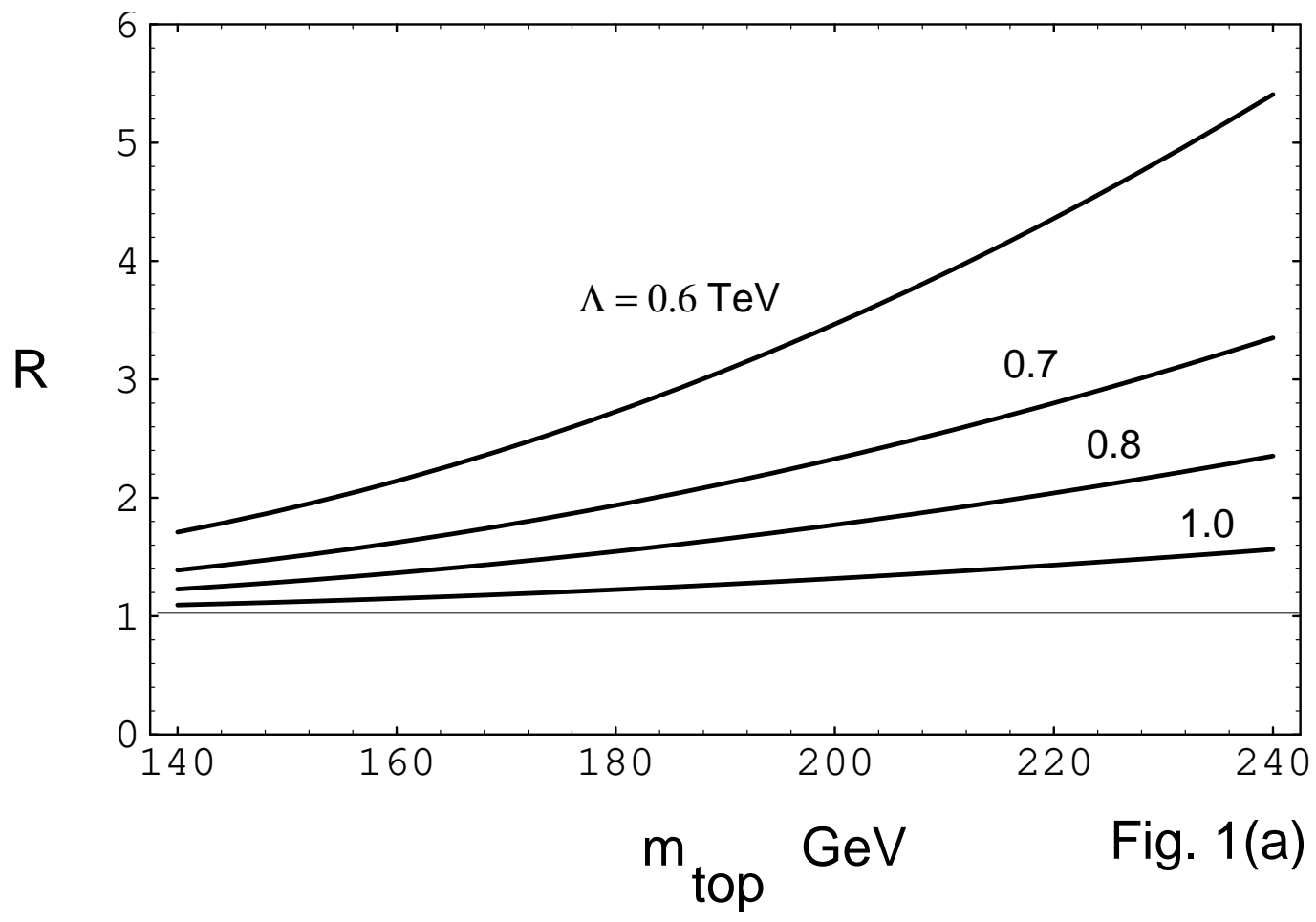




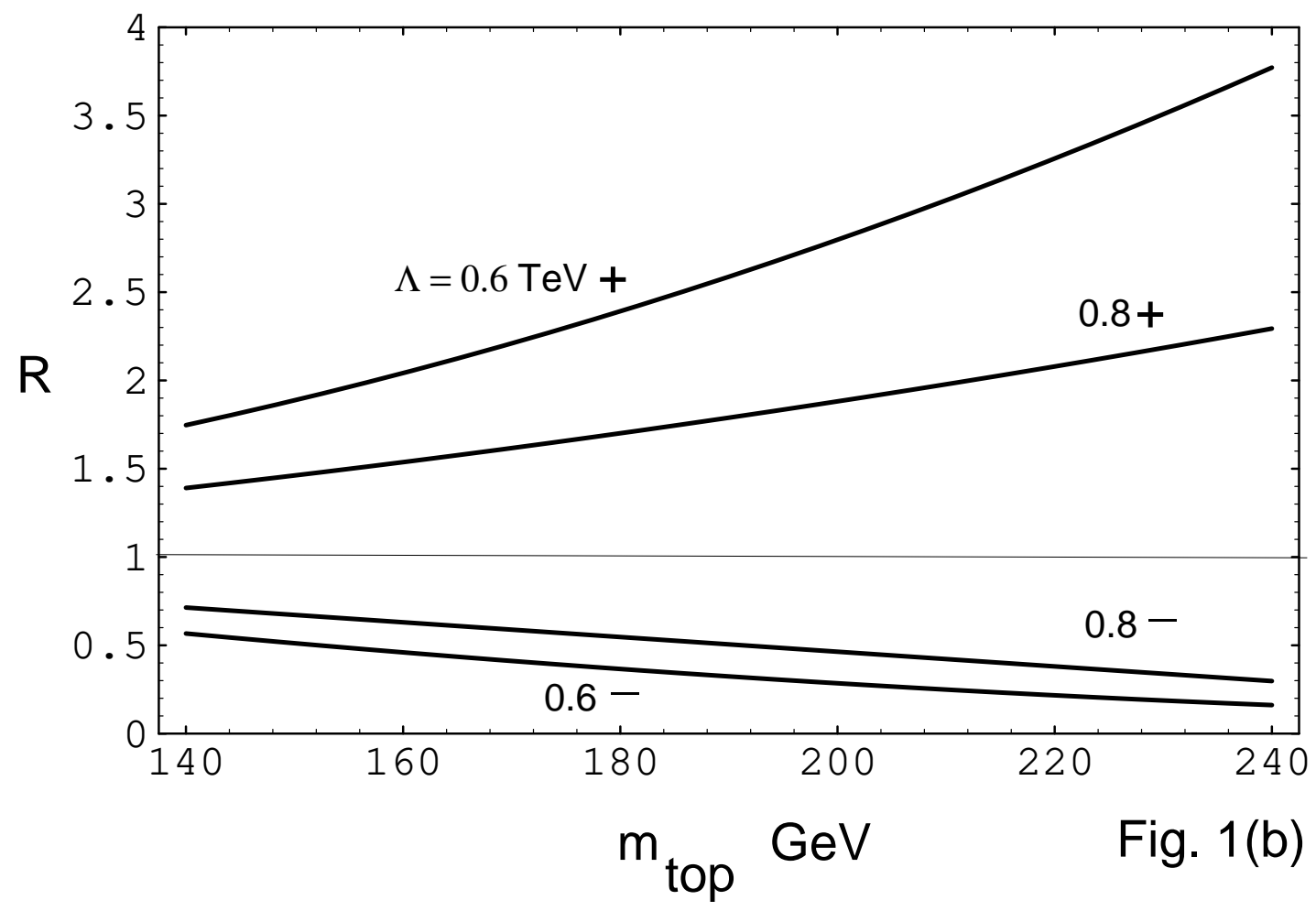


This figure "fig1-2.png" is available in "png" format from: http://arxiv.org/ps/hep-ph/9312324v1 
This figure "fig2-2.png" is available in "png" format from: http://arxiv.org/ps/hep-ph/9312324v1 
This figure "fig3-2.png" is available in "png" format from: http://arxiv.org/ps/hep-ph/9312324v1 


$$
\mathrm{d} \sigma / \mathrm{dP}_{\mathrm{T}}(\mathrm{fb} / \mathrm{GeV})
$$

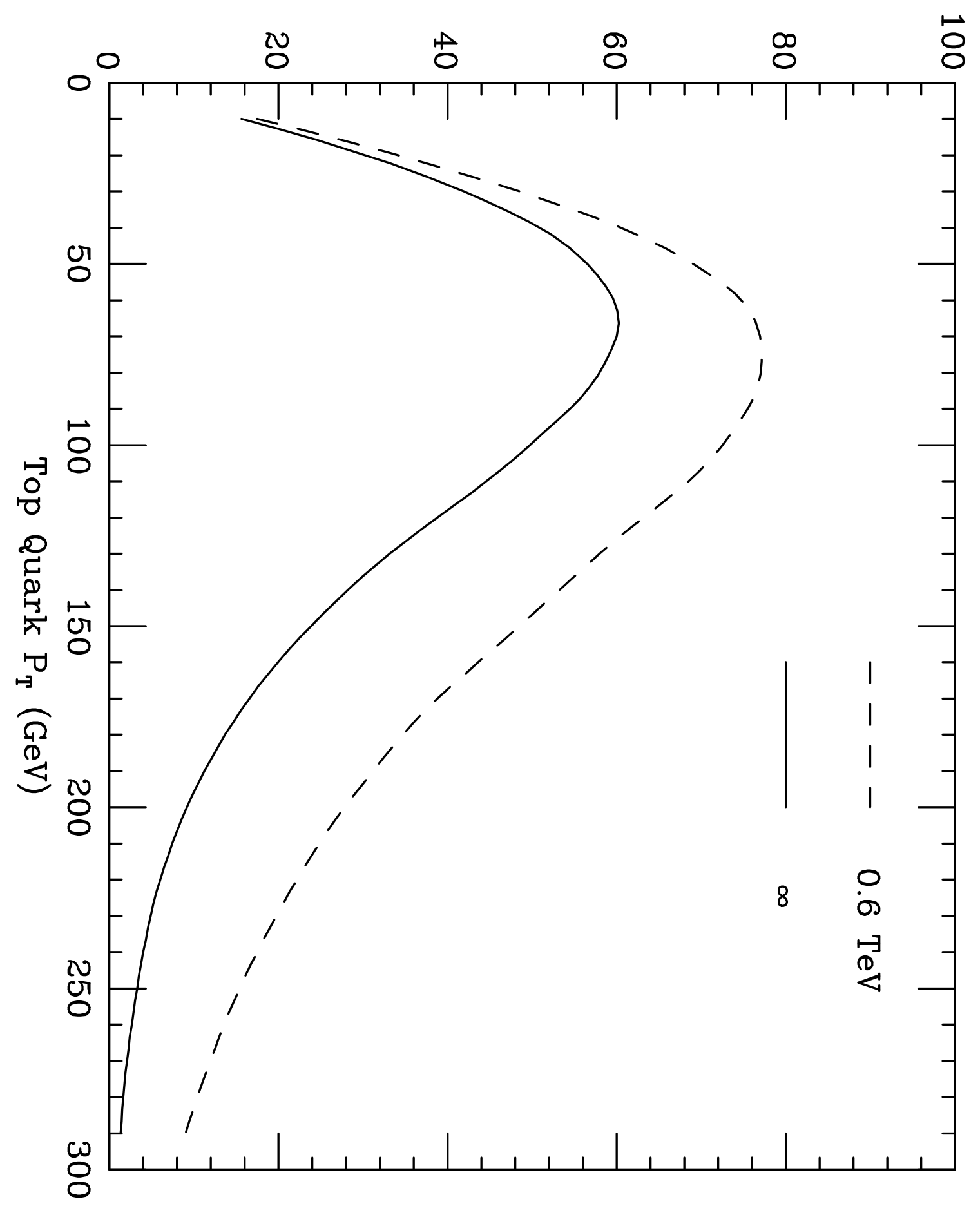

管 
$\mathrm{d} \sigma / \mathrm{dP}_{\mathrm{T}}(\mathrm{fb} / \mathrm{GeV})$

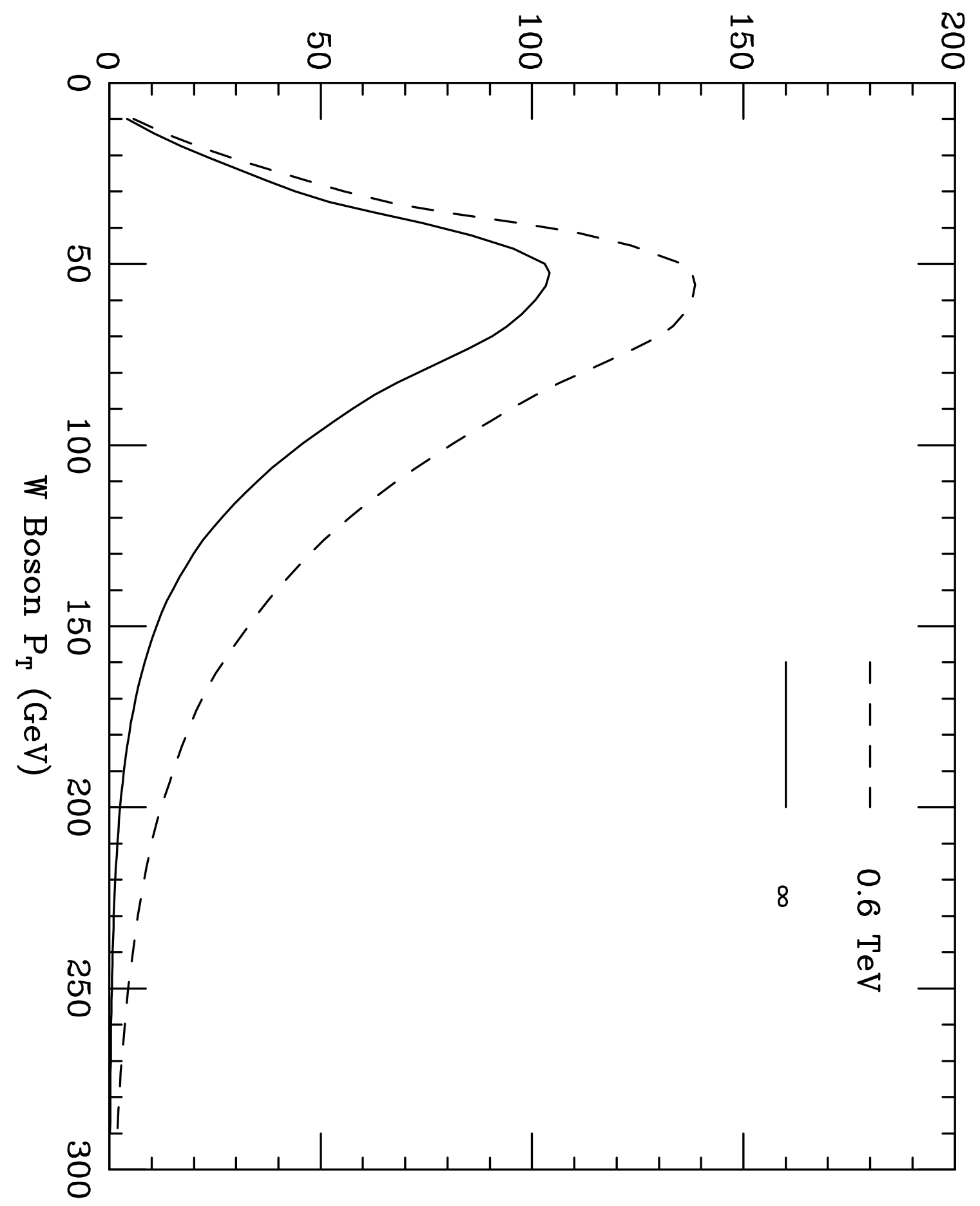

$\underbrace{\infty}_{0}$ 
This figure "fig1-3.png" is available in "png" format from: http://arxiv.org/ps/hep-ph/9312324v1 
This figure "fig2-3.png" is available in "png" format from: http://arxiv.org/ps/hep-ph/9312324v1 
This figure "fig3-3.png" is available in "png" format from: http://arxiv.org/ps/hep-ph/9312324v1 


$$
\mathrm{d} \sigma / \mathrm{dP}_{\mathrm{T}}(\mathrm{fb} / \mathrm{GeV})
$$

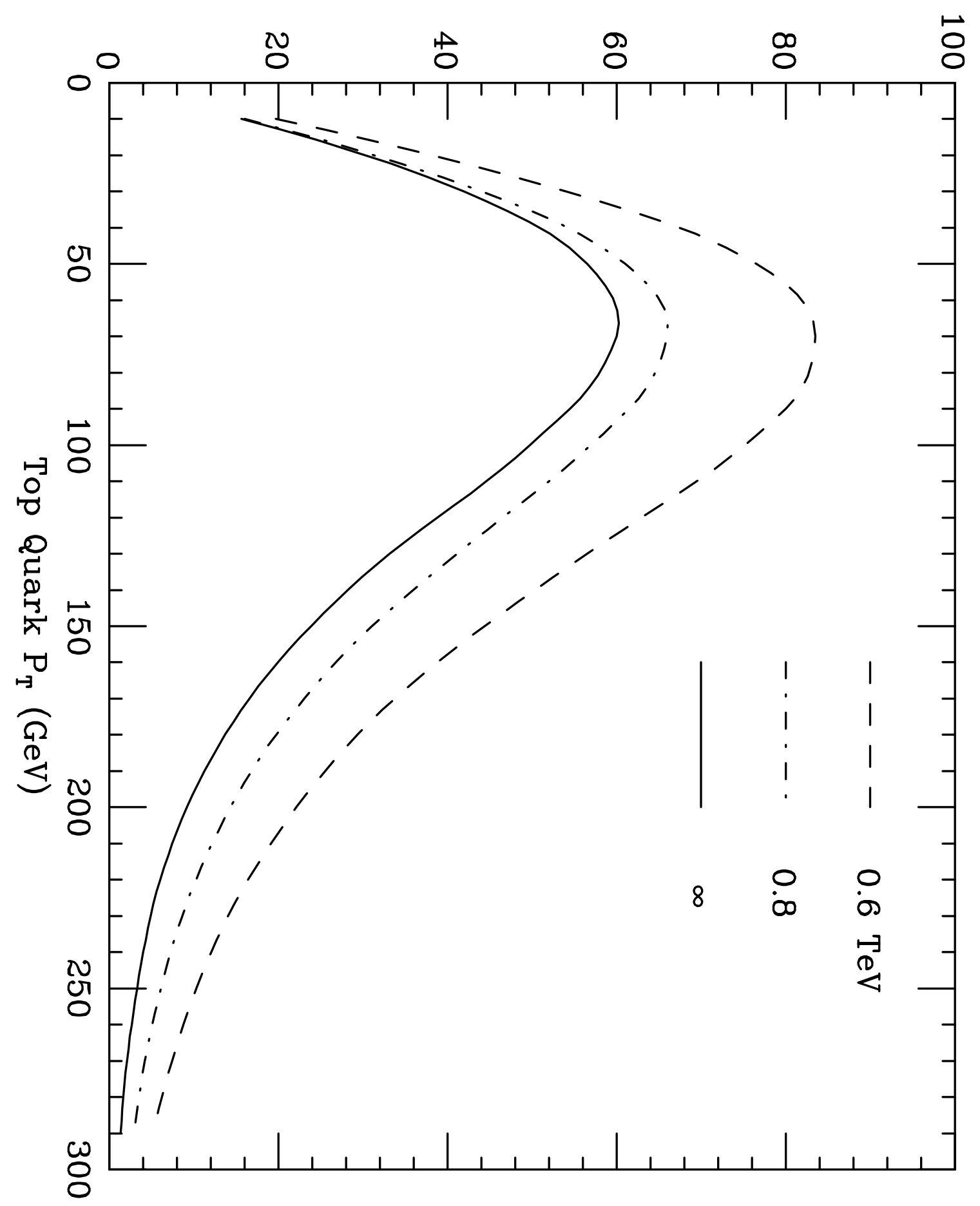

苞 
$\mathrm{d} \sigma / \mathrm{dP}_{\mathrm{T}}(\mathrm{fb} / \mathrm{GeV})$

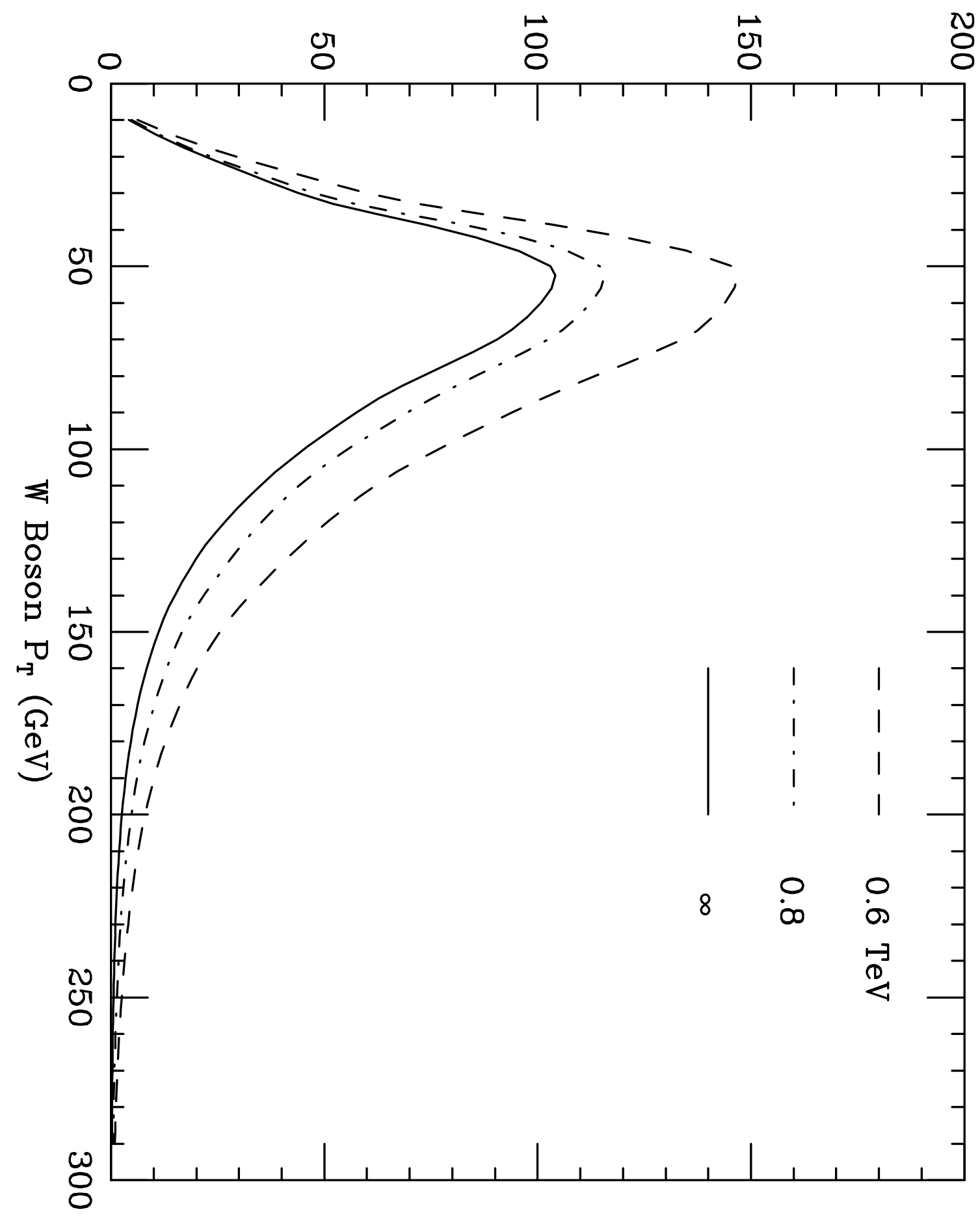

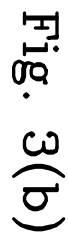


This figure "fig1-4.png" is available in "png" format from: http://arxiv.org/ps/hep-ph/9312324v1 
This figure "fig2-4.png" is available in "png" format from: http://arxiv.org/ps/hep-ph/9312324v1 
This figure "fig3-4.png" is available in "png" format from: http://arxiv.org/ps/hep-ph/9312324v1 


$$
\mathrm{d} \sigma / \mathrm{dP}_{\mathrm{T}}(\mathrm{fb} / \mathrm{GeV})
$$

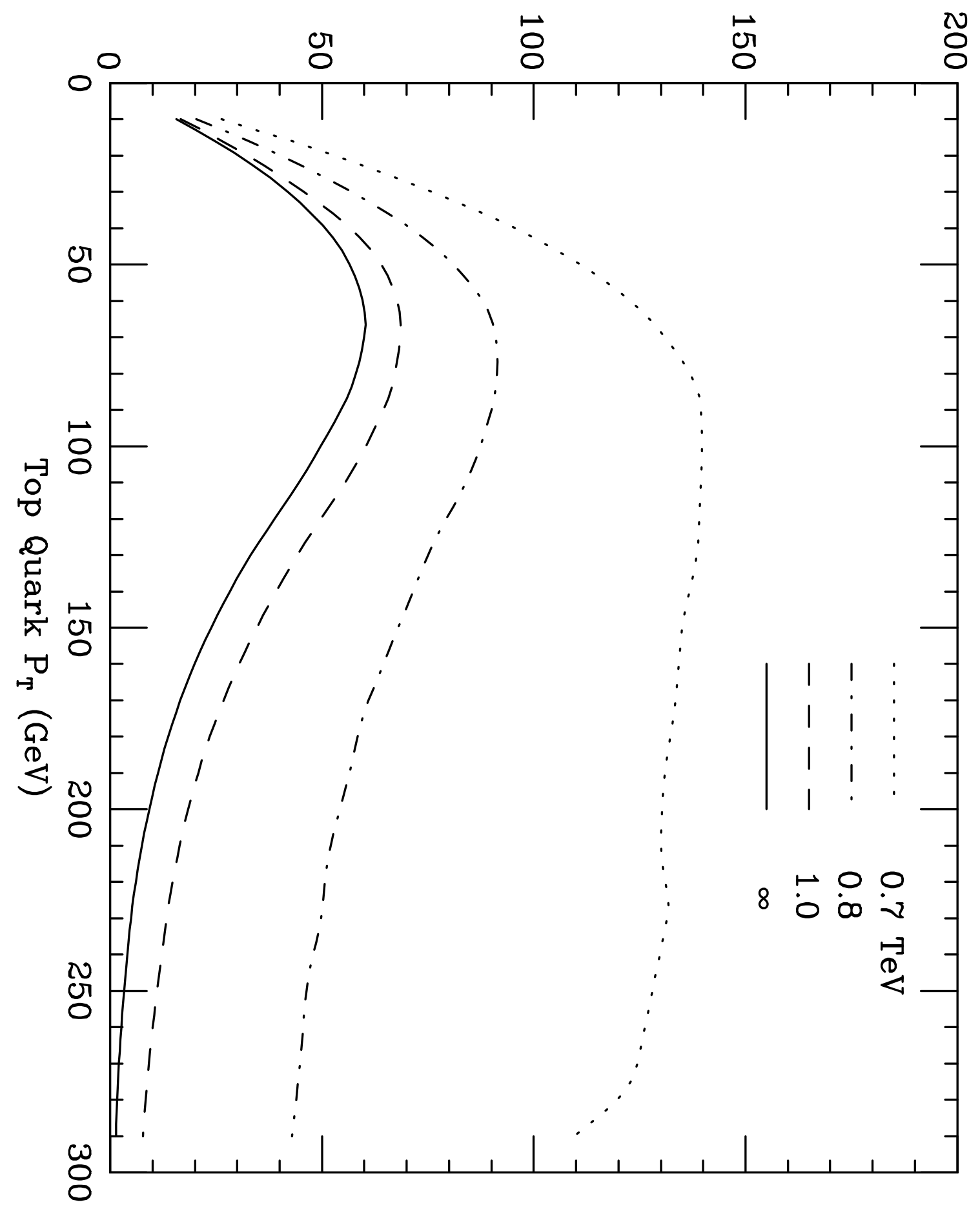

平 
$\mathrm{d} \sigma / \mathrm{dP}_{\mathrm{T}}(\mathrm{fb} / \mathrm{GeV})$

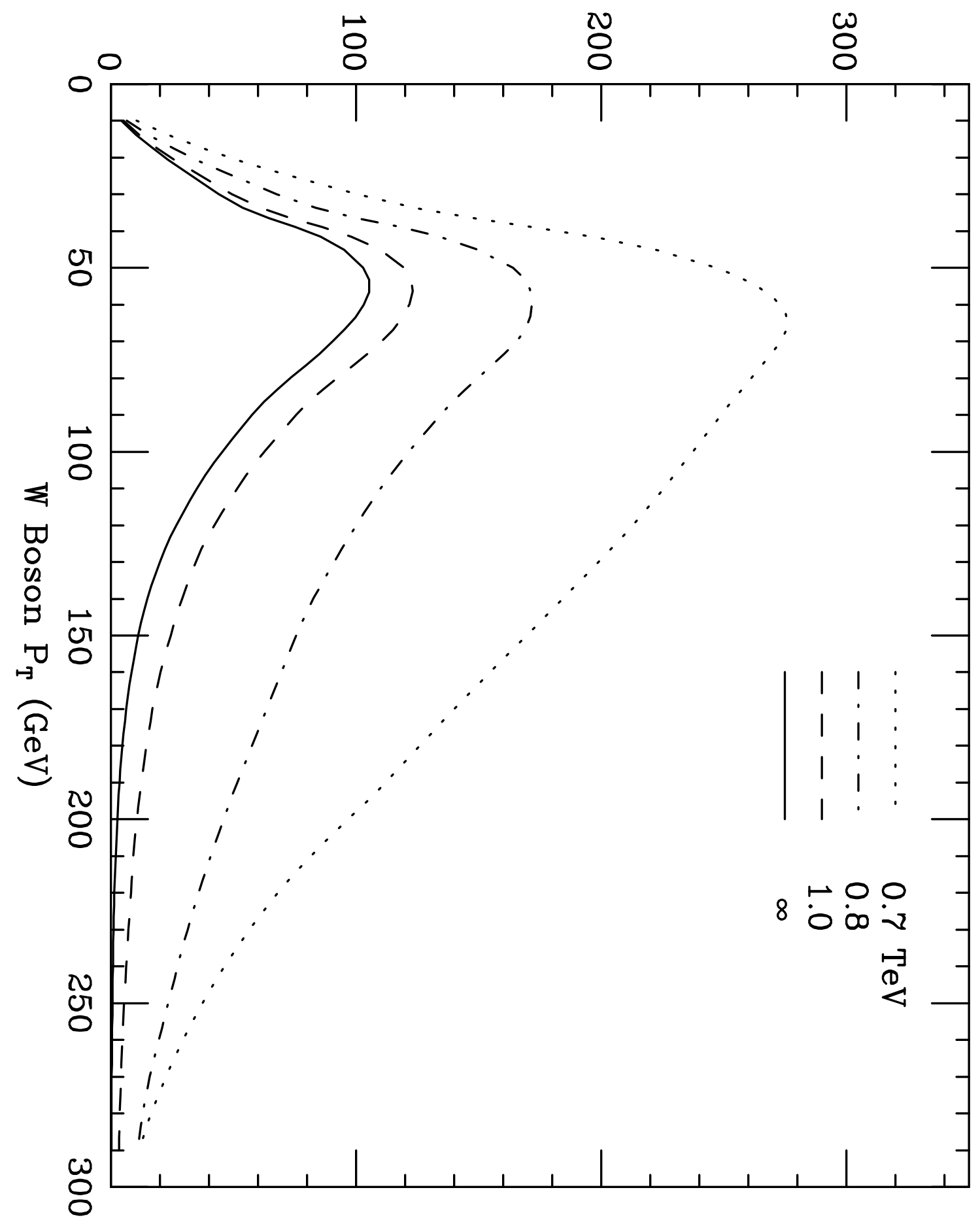

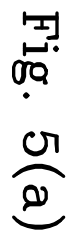




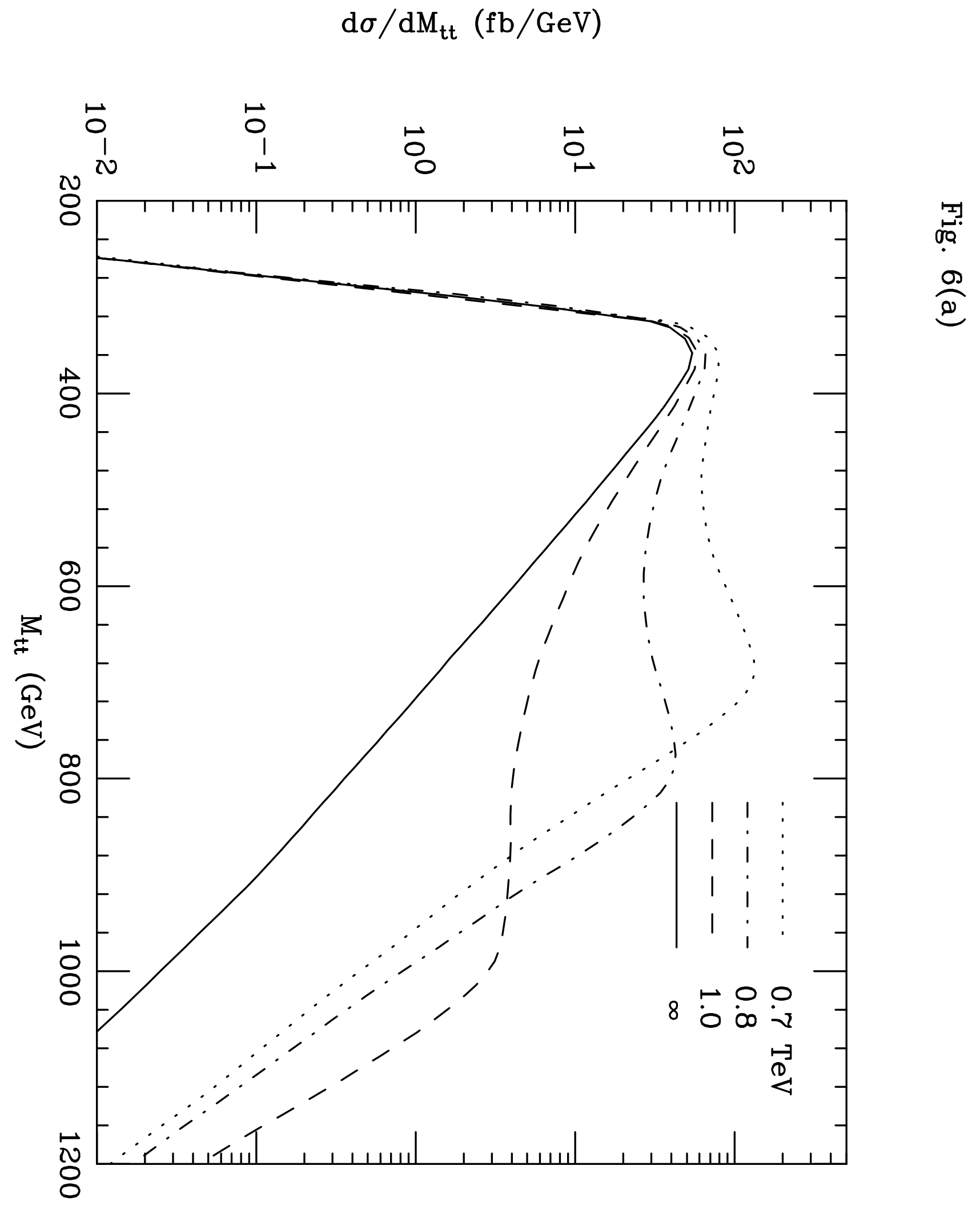


This figure "fig2-5.png" is available in "png" format from: http://arxiv.org/ps/hep-ph/9312324v1 
This figure "fig3-5.png" is available in "png" format from: http://arxiv.org/ps/hep-ph/9312324v1 
$\mathrm{d} \sigma / \mathrm{dP}_{\mathrm{T}}(\mathrm{fb} / \mathrm{GeV})$

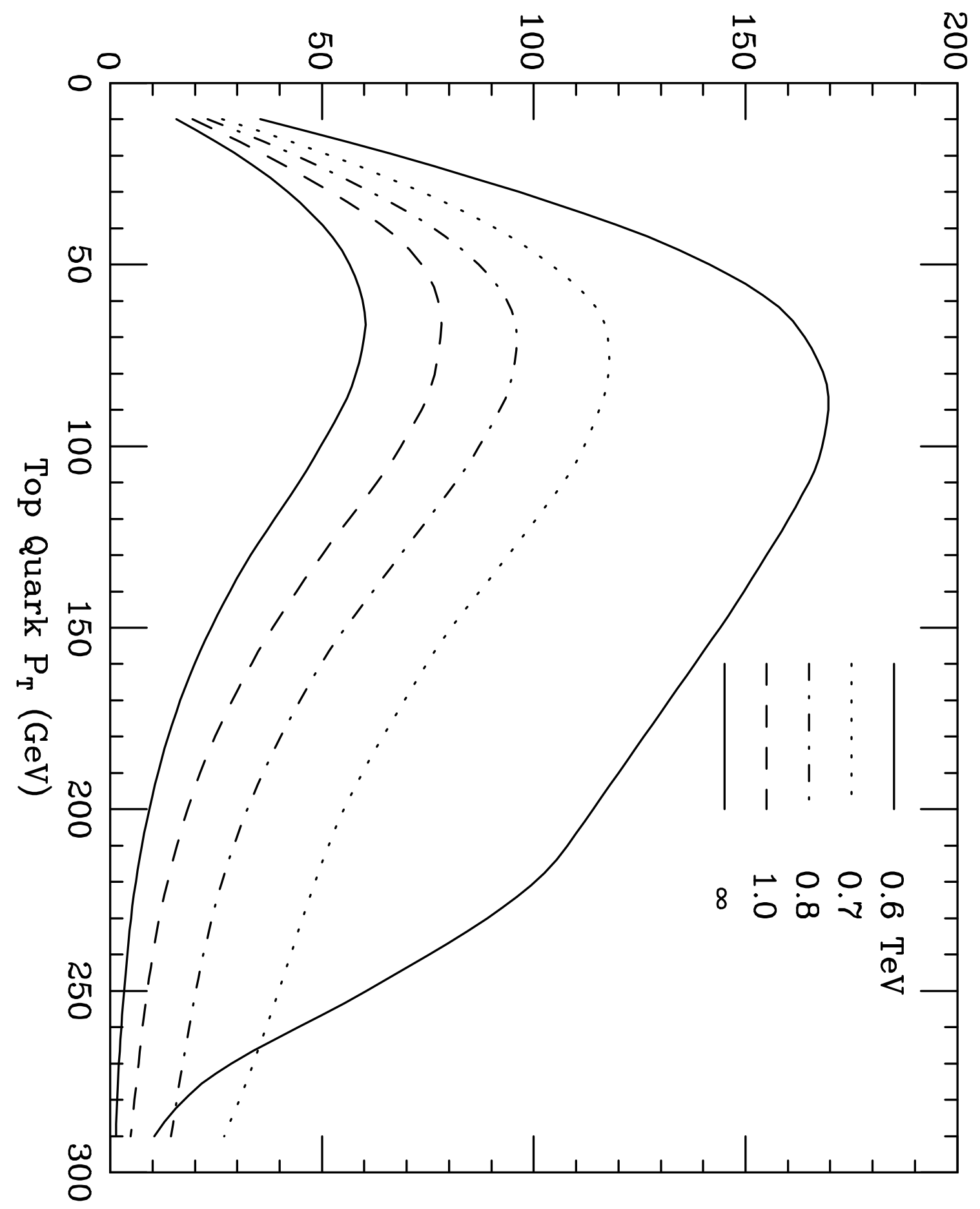

苞 


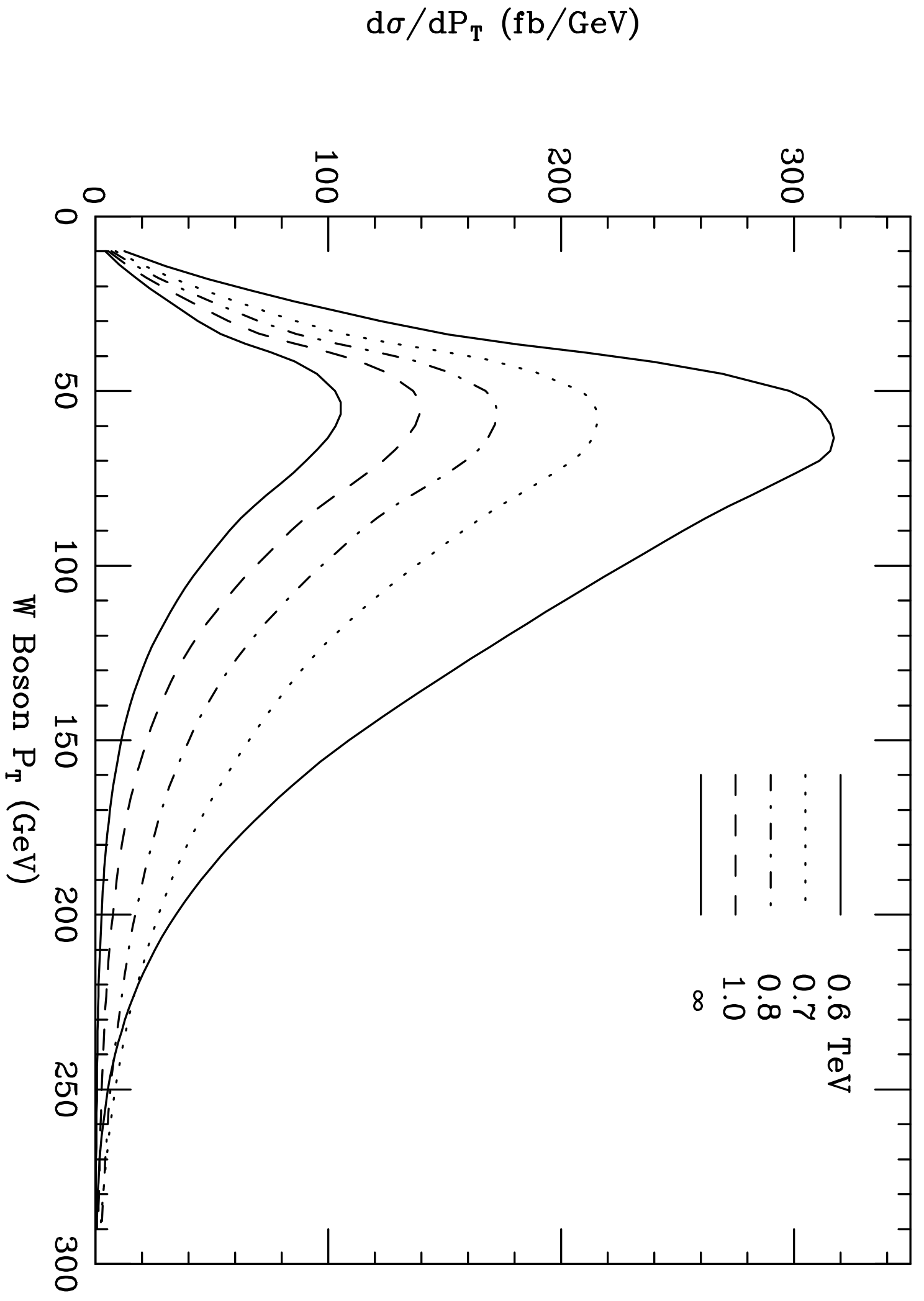

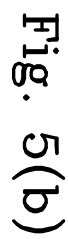




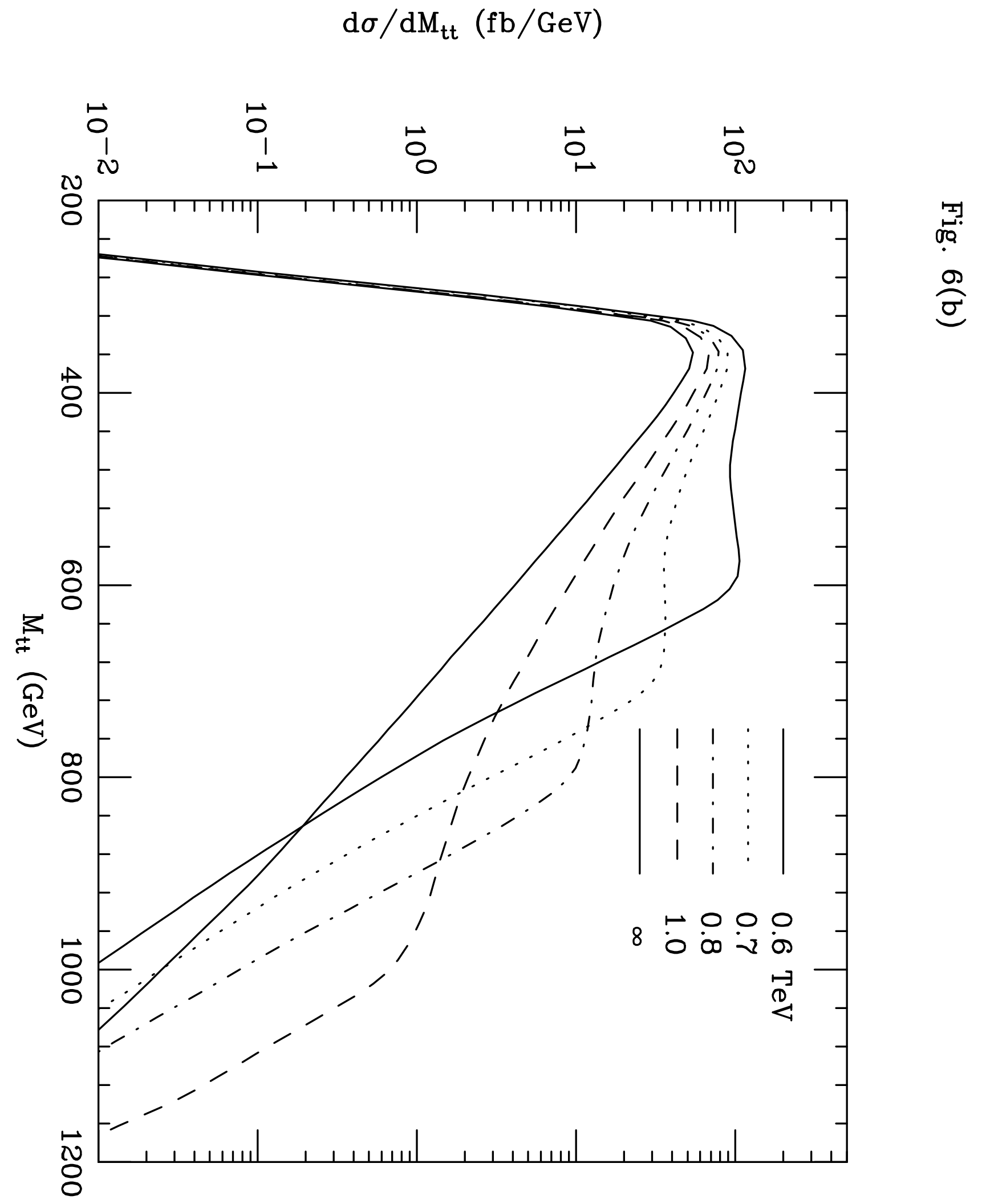


This figure "fig3-6.png" is available in "png" format from: http://arxiv.org/ps/hep-ph/9312324v1 


$$
\mathrm{d} \sigma / \mathrm{dP}_{\mathrm{T}}(\mathrm{fb} / \mathrm{GeV})
$$

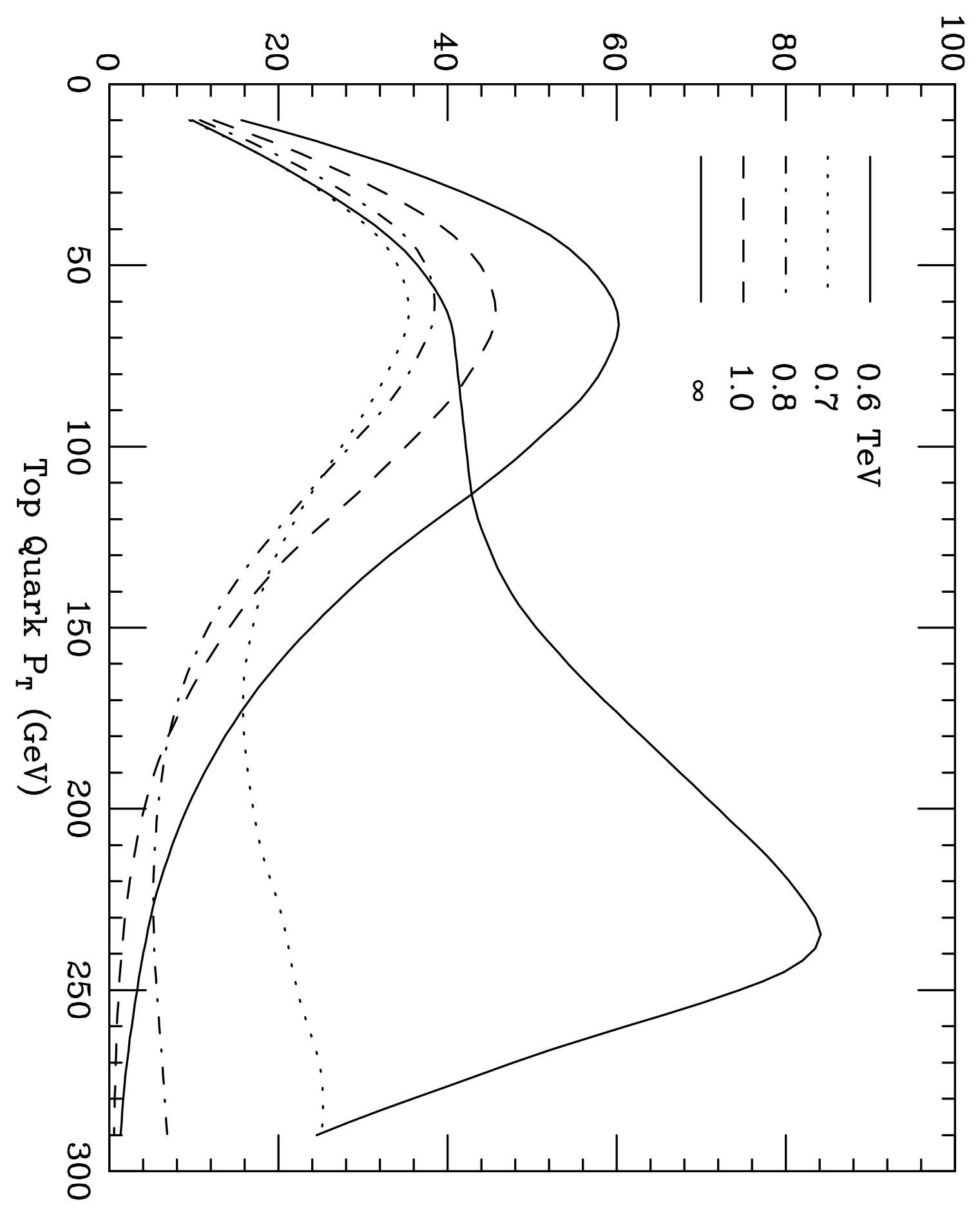

苗 


$$
\mathrm{d} \sigma / \mathrm{dP}_{\mathrm{T}}(\mathrm{fb} / \mathrm{GeV})
$$

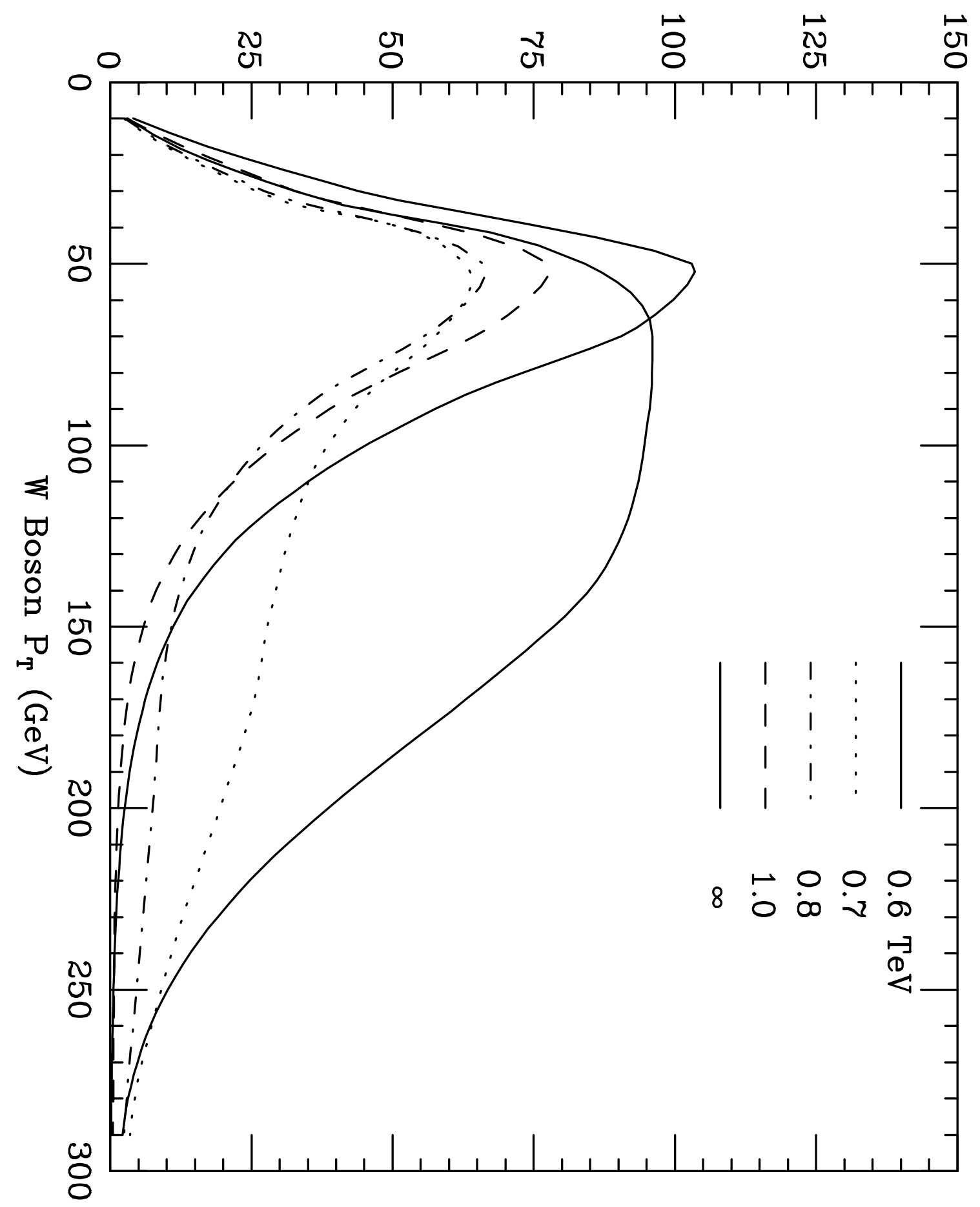

0
$\frac{0}{0}$
0 


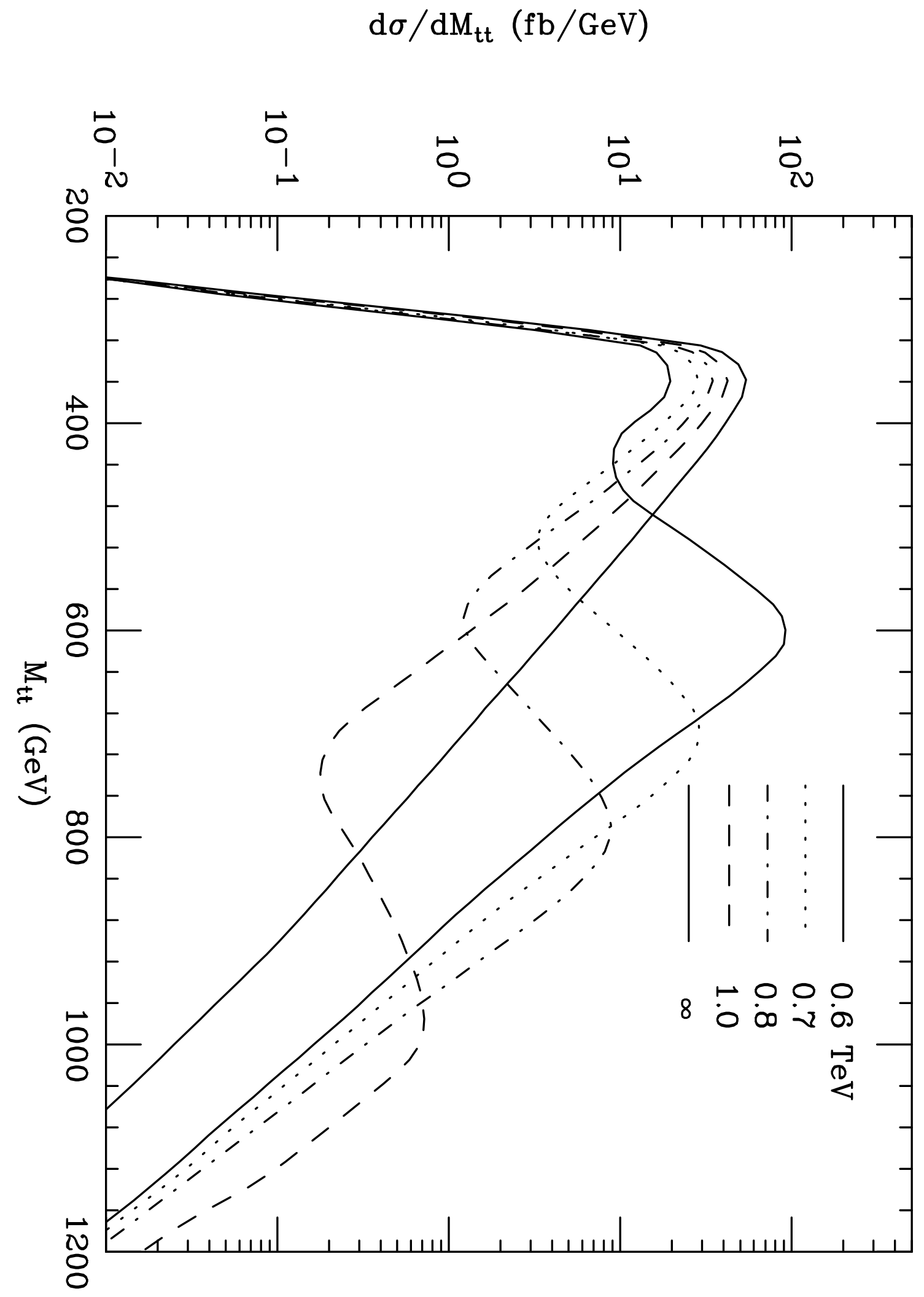

畓 